Curr Treat Options Oncol. 2008 December ; 9(4-6): 277-287. doi:10.1007/s11864-009-0083-7.

\title{
Treatment of Advanced Thymoma and Thymic Carcinoma
}

\author{
Arun Rajan, MD, Giuseppe Giaccone, MD, PhD* \\ Medical Oncology Branch, National Cancer Institute, Bethesda, MD, USA.
}

\section{Opinion statement}

\begin{abstract}
Although thymic epithelial tumors are rare, they are relatively common among neoplasms of the anterior superior mediastinum. They usually exhibit indolent behavior, but do have the capacity to invade surrounding structures and metastasize to distant sites. Thymic carcinomas are rare, but are highly aggressive tumors that are associated with a poor prognosis. The mainstay of therapy is complete surgical resection. Locally advanced thymoma and thymic carcinoma require a multimodality treatment approach with a combination of surgery, chemotherapy, and radiation therapy to decrease the chances of recurrence and improve survival. The risk of disease recurrence lasts for a number of years after completion of primary therapy. A majority of cases of recurrent disease present as pleural recurrences. Once again, surgical resection of recurrent disease represents the cornerstone of successful therapy and is critical to long-term survival. In recent years, a better understanding of the biologic basis of thymic epithelial tumors has led to the emergence of targeted therapy directed against this malignancy.
\end{abstract}

\section{Introduction}

Thymic tumors are amongst the most common mediastinal neoplasms. They comprise about $20 \%$ of all the mediastinal tumors and up to half of all tumors in the anterior mediastinum in adults. However, invasive thymomas and thymic carcinomas are rare tumors and account for $0.2 \%$ to $1.5 \%$ of all the malignancies [1]. Most thymomas are clinically indolent tumors. However, thymic carcinomas are invasive tumors, but account for only about $0.06 \%$ of all thymic neoplasms [2•]. The degree of encapsulation of these thymic tumors and the extent of invasion of surrounding tissue correlate with prognosis [3].

Surgery forms the cornerstone of therapy for early stage disease. For advanced stage or unresectable disease, multimodality therapy is required. Thymomas are sensitive to radiation therapy and chemotherapy.

The aim of this article is to review recent literature that describes the management of thymic epithelial malignancies, with particular emphasis on the treatment of advanced thymoma, thymic carcinoma, and recurrent disease.

*Address giacconeg@mail.nih.gov. 


\section{Staging}

- The most widely used system for staging thymoma was developed by Masaoka in 1981. It was subsequently revised in 1994. The Masaoka system stages the disease based on the degree of tumor encapsulation and the invasion of surrounding structures (Table 1) $[4,5]$.

- $\quad$ The World Health Organization (WHO) introduced a system of classifying thymomas in 1999 that was based on the histological appearance of the tumor [6•].

- A detailed discussion of the histologic classification of thymic malignancies is presented in an article by Suster and Moran [7].

\section{Clinical features}

- $\quad$ Most patients with thymoma are diagnosed between the 5th and 7th decade of life. About two-thirds of patients are asymptomatic at the time of diagnosis. The remaining one-third of patients may develop symptoms, either due to the presence of the underlying mediastinal mass or due to associated immune system disorders. Common symptoms might include chest pain, cough, dyspnea, phrenic nerve paralysis, or the constellation of symptoms associated with the superior vena cava (SVC) syndrome.

- A variety of autoimmune disorders are associated with thymoma. The most common disorder is myasthenia gravis that is seen in $30 \%$ to $65 \%$ of cases $[8,9]$. A number of other neurologic and non-neurologic paraneoplastic syndromes have been described in patients with thymoma [3, 9-11•]. Paraneoplastic diseases associated with thymic malignancies can be potentially put into remission with systemic chemotherapy if the primary tumor responds to chemotherapy, although the data supporting this is scant.

\section{Treatment of early stage thymoma}

- $\quad$ Surgical resection is the mainstay of treatment for patients presenting with Masaoka Stage I or II disease. Resection of the entire tumor is accompanied by complete thymectomy and removal of all surrounding mediastinal fat. Surrounding pleura should also be removed if possible to increase the chances of achieving negative surgical margins. If the tumor abuts the pericardium or an intraoperative frozen section demonstrates invasion of the pericardium, then en bloc resection of the pericardium is recommended. If the patient is thought to be at risk for pleural invasion, the entire pleural space should be explored to exclude the presence of droplet metastases [12••].

- $\quad$ Patients who undergo a complete resection of a stage I tumors have a 5-year survival of $100 \%$ and a recurrence rate of $1 \%$ [13••]. These patients are not considered candidates for adjuvant radiation therapy. For patients with stage II disease, the 5-year survival rate is $98 \%$ and the recurrence rate is about $4 \%$ 
[13••]. Most of these patients might not benefit from postoperative radiation therapy. However patients who are found to have close surgical margins pathologically or intraoperatively, with adjacent organs being within $1 \mathrm{~mm}$ of the tumor, can be considered for adjuvant mediastinal radiation to decrease the chances of local recurrence [12••]. In this context, the WHO subgroup becomes important since a type B tumor might be associated with a higher incidence of local recurrence than a type A tumor [12••].

\section{Treatment of locally advanced thymoma}

- When preoperative evaluation establishes the presence of stage III disease or if patients present with a large tumor mass that is likely to be adherent to adjacent organs, it is not unreasonable to consider induction therapy to increase resectability. Neoadjuvant chemotherapy is associated with objective response rates of $50 \%$ to $80 \%$ when cisplatin-based combination chemotherapy is utilized [14]. Various chemotherapy combinations that have been utilized include cisplatin, doxorubicin and cyclophosphamide (PAC), cisplatin, doxorubicin, vincristine and cyclophosphamide (ADOC), cisplatin, epirubicin and etoposide, and cisplatin, ifosfamide and etoposide (VIP). Response to preoperative chemotherapy may be used to assess potential effects of these drugs in the adjuvant setting.

- If the tumor remains unresectable after neoadjuvant chemotherapy, preoperative radiation therapy can be offered to attempt to further downstage the tumor. Some centers have used concurrent chemoradiotherapy in the neoadjuvant setting to achieve maximal downstaging prior to surgery [15]. However, concomitant chemoradiation is more toxic than chemotherapy alone and may induce significant scarring that renders surgery more challenging.

\section{Adjuvant radiation therapy}

- There is conflicting data on the benefit of postoperative radiation therapy in decreasing the rates of local recurrence after surgical resection of thymoma. It is generally accepted that patients with stage I tumors do not require postoperative radiation therapy after complete resection of the tumor since the recurrence rate is very low. Patients with stage II disease with gross adhesions to the pleura or microscopic pleural invasion are at higher risk for disease recurrence. In a study by Haniuda et al., patients with adhesions to the pleura had recurrence rates of $36.4 \%$ after surgery vs $0 \%$ in patients without adhesions [16]. In the same study the authors reported a relapse rate of $23 \%$ and $30 \%$ with postoperative radiation therapy and $25 \%$ and $25 \%$ without postoperative radiation therapy in completely resected stage II and III thymoma, respectively. In another large series of 1320 patients with thymic epithelial tumors, postoperative radiation therapy failed to demonstrate any significant benefit in reducing recurrence rates in completely resected stage II and III thymoma (4.7\% and $23 \%$ with radiation therapy vs $4.1 \%$ and $26 \%$ without radiation therapy) [13••]. Therefore, postoperative radiation 
therapy may not play a significant role in decreasing recurrence rates in completely resected stage II and III thymoma. However, postoperative radiation therapy can be considered in patients with incompletely resected stage II or III disease.

\section{Treatment of advanced stage thymoma (Stages III and IV)}

- Multiagent chemotherapy inclusive of cisplatin plays an important role in treatment of advanced stage disease. A study conducted by the Southeastern Cancer Study Group was designed to evaluate the combination of cisplatin, doxorubicin, and cyclophosphamide (PAC) in patients with unresectable or advanced stage disease who received up to 6 cycles of PAC. The overall response rate (ORR) was $50 \%$ and median survival was 38 months [17••]. The combination of doxorubicin, cisplatin, vincristine, and cyclophosphamide (ADOC) administered every 4 weeks, yielded an overall response rate of $92 \%$ and median survival of 15 months after a median of 5 cycles of therapy [18•]. The combination of cisplatin and etoposide was tried in 16 patients with recurrent or metastatic thymoma and was associated with an overall response rate of 56\% and median survival of 4.3 years [19•]. Chemotherapy with ifosfamide, etoposide, and cisplatin (VIP) in 28 patients with advanced thymoma or thymic carcinoma yielded a partial response rate of $32 \%$ and an overall survival of 32 months [20]. This study for the first time included a significant number of thymic carcinomas.

- The combination of cisplatin, doxorubicin, and methylprednisolone (CAMP) has been used as part of multimodality therapy in patients with advanced invasive thymoma. In this trial, 14 out of 17 patients received CAMP induction chemotherapy and an overall response rate of $93 \%$ was noted. The 5-year overall survival rate for all the 17 patients in this trial was $81 \%$ [21•].

- Lemma et al. recently reported results of a phase II study of carboplatin with paclitaxel in the cases of advanced thymoma and thymic carcinoma [22]. Patients with unresectable thymomas or thymic carcinoma who had not received prior systemic chemotherapy were eligible. Treatment consisted of carboplatin AUC5 plus paclitaxel $225 \mathrm{mg} / \mathrm{m}^{2}$ administered every 3 weeks up to a maximum of 6 cycles of therapy. Three CRs and five PRs (ORR 33\%) and 15 stable disease (SD) were observed in 24 patients with thymoma and no CR, five PRs (ORR $24 \%$ ) and seven SDs were observed in 21 patients with thymic carcinoma. A progression-free survival (PFS) of 19.8 months and 6.2 months was reported by the authors for thymoma and thymic carcinoma, respectively. Median survival had not been reached in patients with thymoma at the time the study was reported. In patients with thymic carcinoma the median survival was 15 months. The authors concluded that the combination of carboplatin and paclitaxel showed modest clinical benefit in patients with thymic malignancies.

- Table 2 summarizes some of the chemotherapy combinations used in the treatment of invasive thymomas. These results illustrate the role of platinum 
compounds in the treatment of advanced thymic malignancies. It should be noted that although case reports and retrospective analyses have reported response rates of up to $43 \%$ with single agent cisplatin [23], a prospective phase II study of cisplatin reported a low response rate of $10 \%$ in 18 patients treated [24•]. However, multiple studies have shown that cisplatin-based combination chemotherapy is associated with response rates of $50 \%$ to $100 \%$ as illustrated in Table 2. Most of the platinum-based combinations also contain doxorubicin (PAC, ADOC, CAMP). Hence a chemotherapy regimen such as PAC or ADOC can be administered as a component of multimodality therapy for advanced thymic malignancies.

\section{The role of biologic agents}

- $\quad$ Recent studies have focused on the role of Epidermal Growth Factor Receptor (EGFR) and c-KIT expression in thymic tumors [25-27]. It has been shown that thymomas generally express EGFR but lack c-KIT expression whereas the converse is true for thymic carcinomas. Based on these data, a few case reports have documented clinical responses to treatment with biologic agents like cetuximab [28, 29]. In 2005, Kurup et al. had reported results of a phase II study with gefitinib in patients with advanced thymic malignancies [30]. In this study, 26 previously treated patients were enrolled and treated with gefitinib $250 \mathrm{mg}$ orally once a day. Each cycle lasted 28 days and based on response, patients were treated up to a maximum of 8 cycles of therapy. EGFR mutation analysis was performed on five tumor blocks. No CR, 1 PR, and 14 SD were noted. None of the tumor blocks subjected to genomic analyses demonstrated presence of EGFR or KRAS mutations. The authors concluded that gefitinib showed minimal activity in this cohort of patients. Another phase II study reported earlier this year by Bedano and colleagues evaluated the combination of erlotinib and bevacizumab in patients with recurrent thymoma and thymic carcinoma [31]. According to this study, 18 previously treated patients received erlotinib $150 \mathrm{mg}$ orally once a day and bevacizumab $15 \mathrm{mg} / \mathrm{kg}$ intravenously once, with the cycle repeated every 3 weeks. No CRs or PRs and 11 (60\%) were noted; 7 (40\%) patients progressed (PD). The median survival time had not been reached when the results were reported. The combination of erlotinib and bevacizumab demonstrated limited activity in patients with thymic malignancies.

- A case report published in 2004 documented response of a thymic carcinoma with c-KIT overexpression and an activating mutation of the KIT gene, to imatinib [32•]. Based on the knowledge that c-KIT is highly expressed in thymic carcinomas (80\%), a phase II study was conducted to evaluate the role of imatinib in patients with WHO B3 and C thymomas [33]. Seven patients were enrolled with a median age of 67 years. Two patients had WHO B3 and five patients had WHO C thymoma. Three patients had not received prior chemotherapy. Imatinib was administered at a dose of $600 \mathrm{mg}$ per day orally. The dose was escalated to $800 \mathrm{mg}$ per day for PD or if no major response was achieved after 2 months of therapy. No objective responses were noted. Two 
patients showed stable disease and five patients progressed. Median time to progression was 2 months and median survival was 4 months. Five tumor samples were subjected to genomic analyses. No activating mutations of c-KIT were detected, and this probably explained the disappointing results associated with imatinib therapy. Salter et al. reported a series of 11 patients with previously treated thymic carcinoma expressing c-KIT ( 9 cases) and PDGFR ( 2 cases) detected by immunohistochemistry [34]. These patients were treated with imatinib $600 \mathrm{mg}$ by mouth once daily. No objective responses were noted although three patients achieved disease stabilization.

- These studies appear to demonstrate the lack of significant activity of imatinib in patients with thymic carcinoma. A case of a dasatinib-responsive thymoma has also been documented in the literature. In this case, the patient who was initially diagnosed and treated for chronic myelogenous leukemia, was found to have a WHO B2, Masaoka Stage I thymic tumor that was EGFR positive and c-KIT negative. After 2 months of therapy with dasatinib at a dose of $140 \mathrm{mg} / \mathrm{day}$, the thymic tumor showed partial resolution [35].

\section{Thymic carcinoma}

- Thymic carcinomas differ from thymomas in that they follow an aggressive clinical course and are associated with a poorer prognosis. These tumors show a variety of histologic patterns based on which they have been classified as lowgrade or high-grade tumors. Low-grade thymic carcinomas include squamous cell carcinoma, muco-epidermoid carcinoma, and basaloid carcinoma. Highgrade varieties include lymphoepithelioma-like carcinoma, small cell carcinoma, sarcomatoid tumors, clear cell carcinomas, and undifferentiated tumors [36••]. Thymic carcinomas are rarely associated with paraneoplastic syndromes. Metastatic disease may be seen at the time of presentation, and the sites of involvement include the bones, lung, liver, pleura, and lymph nodes. At the molecular level thymic carcinomas frequently show expression of c-KIT but are less likely to be EGFR positive in contrast to thymomas where the converse is true. p53 expression and chromosomal imbalances are seen more often in thymic carcinomas as opposed to thymomas, and correlate with the WHO class and stage of the disease [37•-39].

- In 1993, Weide et al. reported the results of a small series of five patients with thymic carcinoma treated with cisplatin-based combination chemotherapy. The chemotherapy regimen used included cisplatin, etoposide and belomycin (BEP), cisplatin, vinblastine and ifosfamide, cisplatin/carboplatin and ifosfamide, cisplatin, vinblastine and bleomycin, and cisplatin, doxorubicin and cyclophosphamide (PAC). Three out of five patients responded with two complete responses to cisplatin-based combination chemotherapy [40•].

- Multimodality therapy is often the preferred approach for treatment of thymic carcinomas, which frequently are not radically resectable at diagnosis (Table 3 ). Complete resection of the tumor is a very important prognostic factor for survival [41]. Use of neoadjuvant chemotherapy with or without radiation therapy 
improves the rates of complete surgical resection [2•]. Table 3 summarizes case series that highlight the role of combined modality therapy in the management of thymic carcinoma.

\section{Treatment of recurrent disease}

- The recurrence rate of thymoma after complete surgical resection ranges from $10 \%$ to $30 \%$. The most common site of recurrence is the pleural cavity followed by the mediastinum. An early recurrence is considered a negative prognostic factor [45]. Surgery may be considered in patients who are radically resectable, although data in the literature also supports a potential role for debulking surgery. Various forms of chemotherapy and biologic therapy have been used to treat recurrent disease (Table 4). An ECOG phase II trial was conducted to evaluate the role of octreotide in patients with octreotide-scan positive thymic malignancies. Patients with pretreatment octreotide-scan positive unresectable or advanced thymoma, and thymic carcinoma were selected. Out of 38 assessable patients, 32 had thymoma and six patients had thymic carcinoma or carcinoid; 35 patients (92\%) had undergone surgery, 32 (84\%) had received prior radiation therapy, and $31(82 \%)$ had been exposed to prior chemotherapy. Out of 38 patients, 34 had Masaoka stage IV disease. In the assessable patients, two complete (CRs) and 10 partial responses (PRs) were noted yielding complete and partial response rates of 5.3\% and 25\%, respectively. The 2-year survival rate was $76 \%$. However, none of the six patients with thymic carcinoma or carcinoid had an objective response to therapy. Among those patients who received a combination of octreotide and prednisone, the overall response rate was noted to be slightly higher at $31.6 \%$, but with increased toxicity [46].

- $\quad$ Results of a phase II study evaluating the role of pemetrexed in patients with recurrent thymoma and thymic carcinoma were reported in 2006 [47•]. In this trial, 16 patients with recurrent thymoma and 11 patients with thymic carcinoma participated. The median number of prior therapies was 2 , and 21 patients had received prior radiation therapy. In 23 evaluable patients, two complete responses and two partial responses were noted. All four responding patients had stage IVA thymoma. The median time to progression for patients with recurrent thymoma was 45.4 weeks, and in patients with thymic carcinoma it was 5.1 weeks. This study demonstrated the activity of pemetrexed in patients with recurrent thymoma.

- $\quad$ Response of thymic malignancies to paclitaxel and docetaxel has also been reported in the literature [48, 49].

- The pleural cavity is a common site of recurrence of thymic malignancies, and attempts have been made toward extensive surgery (extrapleural pneumonectomy). A recent review by Lucchi et al. focused on surgical treatment of pleural recurrences of thymoma [50•]. In case of large pleural recurrences, the authors recommend multimodality treatment with the use of corticosteroids prior to surgery. For those patients who have a residual mass after surgery, low-dose 
entire hemithorax radiation therapy (EHRT) and intraoperative hyperthermic intrathoracic perfusion chemotherapy (HITHOC) has been attempted [51, 52].

- Similar to other tumor types re-resection can be considered if the time to relapse is long.

\section{Conclusions}

- $\quad$ Patients with stage I thymoma should be treated with surgical excision of the tumor. Some patients with stage II disease derive benefit with the addition of postoperative radiation therapy. However, for advanced stage thymoma (stages III and IV) and thymic carcinoma, multimodality therapy plays a key role in successful management of disease. Neoadjuvant chemotherapy is delivered to make unresectable tumors resectable, and to increase the chances of performing complete surgical excision of the tumor. Various chemotherapeutic regimens have been developed, and most of them include cisplatin and doxorubicin. Recent attempts to incorporate the use of biologic agents have not proven successful so far. EGFR inhibition with gefitinib and c-KIT inhibition with imatinib has been attempted, but these drugs appear to lack substantial activity against advanced thymic malignancies.

- In conclusion it is recommended that patients with these rare tumors be enrolled on prospective studies to develop more efficacious therapies against thymic malignancies.

\section{References and Recommended Reading}

Papers of particular interest, published recently, have been highlighted as:

- Of importance

•• Of major importance

1. Fornasiero A, Daniele O, Ghiotto C, et al.: Chemotherapy of invasive thymoma. J Clin Oncol 1990, 8(8):1419-1423. [PubMed: 2380761]

2•. Greene MA, Malias MA: Aggressive multimodality treatment of invasive thymic carcinoma. J Thorac Cardiovasc Surg 2003, 125(2):434-436. doi:10.1067/mtc.2003.133. [PubMed: 12579125]

3. Johnson SB, Eng TY, Giaccone G, Thomas CR: Thymoma: update for the new millennium. The Oncologist 2001, 6:239-246. doi:10.1634/theoncologist.6-3-239. [PubMed: 11423670]

4. Masaoka A, Yamakawa Y, Niwa H, et al.: Follow-up study of thymoma with special reference to their clinical stages. Cancer 1981; 48: 2485-92. doi: 10.1002/1097-0142(19811201)48:11<2485::AIDCNCR2820481123>3.0.CO;2-R. [PubMed: 7296496]

5. Masaoka A, Yamakawa Y, Niwa H, et al.: Thymectomy and malignancy. Eur J Cardiothoracic Surg 1994, 8:251-253. doi:10.1016/1010-7940(94)90155-4.

6•. Okumura M, Onta M, Tateyama H, et al.: The World Health Organization histologic classification system reflects the oncologic behavior of thymoma: a clinical study of 273 patients. Cancer 2002, 94:624-632. doi:10.1002/cncr.10226. [PubMed: 11857293]

7. Suster S, Moran CA: Histologic classification of thymoma: the World Health Organization and beyond. Hematol Oncol Clin N Am 2008, 22:381-392. doi:10.1016/j.hoc.2008.03.001. 
8. Lopez-Cano M, Ponseti-Bosch JM, Espin-Basany E, et al.: Clinical and pathologic predictors of outcome in thymoma-associated myasthenia gravis. Ann Thorac Surg 2003, 76:1643-1649. doi: 10.1016/S00034975(03)01139-1. [PubMed: 14602301]

9. Morgenthaler TI, Brown LR, Colby TV, et al.: Thymoma. Mayo Clin Proc 1993, 68:1110-1123. [PubMed: 8231276]

10. Suadjian JV, Enriquez P, Siverstein MN, et al.: The spectrum of diseases associated with thymoma: coincidence or syndrome? Arch Intern Med 1974, 134:374-379. doi:10.1001/archinte.134.2.374. [PubMed: 4602050]

11. Tormoehlen LM, Pascuzzi RM: Thymoma, myastenia gravis, and other paraneoplastic syndromes. Hematol Oncol Clin North Am 2008, 22(3):509-526. doi:10.1016/j.hoc.2008.03.004. [PubMed: 18514130]

12••. Wright CD: Management of thymomas. Crit Rev Oncol/Hematol 2008, 65:109-120. doi: 10.1016/j.critrevonc.2007.04.005.

13••. Kondo K, Monden Y: Therapy for thymic epithelial tumors: a clinical study of 1320 patients from Japan. Ann Thorac Surg 2003, 76:878-885. doi:10.1016/S0003-4975(03)00555-1. [PubMed: 12963221]

14. Giaccone G, Wilnik H, Paul MA, van der Valk P: Systemic treatment of malignant thymoma. Am J Clin Oncol 2006, 29:336-344. doi:10.1097/01.coc.0000227481.36109.e7. [PubMed: 16891859]

15. Wright CD, Choi NC, Wain JC, et al.: Induction chemoradiotherapy followed by resection for locally advanced Masaoka stage III and IVA thymic tumors. Ann Thorac Surg 2008, 85(2):385389. doi:10.1016/j.athoracsur.2007.08.051. [PubMed: 18222230]

16. Haniuda M, Morimoto M, Nishimura $\mathrm{H}$, et al.: Adjuvant radiotherapy after complete resection of thymoma. Ann Thorac Surg 1992, 54:311-315. [PubMed: 1637226]

17••. Loehrer PJ, Kim KM, Aisner SC, et al.: Cisplatin plus doxorubicin plus cyclophosphamide in metastatic or recurrent thymoma: final results of an Intergroup Trial. J Clin Oncol 1994, 12:1164-1168. [PubMed: 8201378]

18. Fornasiesro A, Daniele O, Ghiotto C, et al.: Chemotherapy for invasive thymoma: A 13 year experience. Cancer 1991; 68:30-33. doi:10.1002/1097-0142(19910701)68:1<30::AIDCNCR2820680106>3.0.CO;2-4. [PubMed: 2049749]

19•. Giaccone G, Ardizzoni A, Kirkpatrick A, et al.: Cisplatin and Etoposide combination chemotherapy for locally advanced or metastatic thymoma: a phase II study of the European Organization for Research and Treatment of Cancer Lung Cancer Cooperative Group. J Clin Oncol 1996, 14:814-820. [PubMed: 8622029]

20. Loehrer PJ, Jiroutek M, Aisner S, et al.: Combined Etoposide, ifosfamide and cisplatin in the treatment of patients with advanced thymoma and thymic carcinoma. An Intergroup Trial. Cancer 2001; 91: 2010-2015. doi:10.1002/1097-0142(20010601)91:11<2010::AID-CNCR1226>3.0.CO; 2-2. [PubMed: 11391579]

21 • Yokoi K, Matsuguma H, Nakahara R, et al.: Multidisciplinary treatment for advanced invasive thymoma with cisplatin, doxorubicin, and methylprednisolone. J Thorac Oncol 2007, 2:73-78. doi:10.1097/JTO.0b013e31802bafc8. [PubMed: 17410014]

22. Lemma GL, Loehrer PJ, Lee JW, et al.: A phase II study of carboplatin plus paclitaxel in advanced thymoma or thymic carcinoma: E1C99. J Clin Oncol 2008, ASCO Annual Meeting Proceedings; 26 [Abstract 8018].

23. Hejna M, Haberl I, Raderer M: Nonsurgical management of malignant thymoma. Cancer 1999, 85:1871-1884. [PubMed: 10223225]

24•. Bonomi PD, Finkelstein D, Aisner S, Ettinger D: EST 2582 phase II trial of cisplatin in metastatic or recurrent thymoma. Am J Clin Oncol 1993, 16(4):342-345. doi: 10.1097/00000421-199308000-00014. [PubMed: 8328413]

25. Henley JD, Cummings OW, Loehrer PJ Sr: Tyrosine kinase receptor expression in thymomas. J Cancer Res Clin Oncol 2004, 130:222-224. doi:10.1007/s00432004-0545-z. [PubMed: 14762710]

26•. Meister M, Schirmacher P, Dienemann H, et al.: Mutational status of the epidermal growth factor receptor (EGFR) gene in thymomas and thymic carcinomas. Cancer Lett 2007, 248:186-191. doi:10.1016/j.canlet.2006.07.003. [PubMed: 16919868] 
27. Nakagawa K, Matsuno Y, Kunitoh H, et al.: Immunohistochemical KIT (CD117) expression in thymic epithelial tumors. Chest 2005, 128:140-144. doi:10.1378/chest.128.1.140. [PubMed: 16002927]

28. Farina G, Garassino MC, Gambacorta M, et al.: Response of thymoma to cetuximab. Lancet Oncol 2007, 8:449-450. doi:10.1016/S14702045(07)70141-9. [PubMed: 17466903]

29. Palmieri G, Marino M, Salvatore M, et al.: Cetuximab is an active treatment of metastatic and chemorefractory thymoma. Front Biosci 2007, 12:757-761. doi:10.2741/2098. [PubMed: 17127335]

30. Kurup A, Burns M, Dropcho S, et al.: Phase II study of gefitinib treatment in advanced thymic malignancies. J Clin Oncol 2005, ASCO Annual Meeting Proceedings; 23: [Abstract 7068].

31. Bedano PM, Perkins S, Burns M, et al.: A phase II trial of erlotinib plus bevacizumab in patients with recurrent thymoma or thymic carcinoma. J Clin Oncol 2008, ASCO Annual Meeting Proceedings; 26: [Abstract 19087].

32-. Strobel P, Hautmann M, Jakob A, et al.: Thymic carcinoma with overexpression of mutated KIT and the response to imatinib. N Eng J Med 2004, 350:2625-2626. doi:10.1056/ NEJM200406173502523.

33. Giaccone G, Smit EF, van Groeningen C, Hogedoorn PC. Phase II study of imatinib in patients with WHO B3 and C thymomas. J Clin Oncol 2008, ASCO Annual Meeting Proceedings; 26 : [Abstract 14665].

34. Salter JT, Lewis D, Yiannoutsos C, et al.: Imatinib for the treatment of thymic carcinoma. J Clin Oncol 2008, ASCO Annual Meeting Proceedings; 26: [Abstract 8116].

35. Chuah C, Lim TH, Lim AST, et al.: Dasatinib induces a response in malignant thymoma. J Clin Oncol 2006, 24:e56-e58. doi:10.1200/JCO.2006.08.8963. [PubMed: 17135636]

36••. Suster S, Rosai J: Thymic carcinoma: a clinicopathologic study of 60 cases. Cancer 1991; 67: 1025-1032. doi:10.1002/1097-0142(19910215)67:4<1025::AIDCNCR2820670427>3.0.CO;2-F. [PubMed: 1991250]

37•. Eng TY, Fuller CD, Jagirdar J, et al.: Thymic carcinoma: state of the art review. Int J Radiat Oncol Biol Phys 2004, 59:654-664. doi:10.1016/j.ijrobp.2003.11.021. [PubMed: 15183468]

38. Penzel R, Hoegel J, Schmitz W, et al.: Clusters of chromosomal imbalances in thymic epithelial tumors are associated with the WHO classification and the staging system according to Masaoka. Int J Cancer 2003, 105:494-498. doi:10.1002/ijc.11101. [PubMed: 12712440]

39. Inoue M, Starostik P, Zettl A, et al.: Correlating genetic abnormalities with World Health Organization-defined histology and stage across the spectrum of thymomas. Cancer Res 2003, 63:3708-3715. [PubMed: 12839963]

40•. WeideLG Ulbright TM, Loehrer PJ Sr, Williams SD: Thymic carcinoma. A distinct clinical entity responsive to chemotherapy. Cancer 1993;71(4): 1219-1223. doi: 10.1002/1097-0142(19930215)71:4<1219::AID-CNCR2820710408>3.0.CO;2-B. [PubMed: 8435796]

41. Lucchi M, Mussi A, Basolo F, et al.: The multimodality treatment of thymic carcinoma. Eur J Cardiothorac Surg 2001, 19:566-569. doi:10.1016/S10107940(01)00666-2. [PubMed: 11343932]

42. Magois E, Guigay J, Blancard PS, et al.: Multimodal treatment of thymic carcinoma: report of nine cases. Lung Cancer 2008, 59:126-132. doi:10.1016/j.lungcan.2007.05.016. [PubMed: 17614156]

43•. Hernandez-Ilizaliturri FJ, Tan D, Cipolla D, et al.: Multimodality therapy for thymic carcinoma (TCA). Results of a 30-year single-institution experience. Am J Clin Oncol 2004, 27:68-72. doi: 10.1097/01.coc.0000046301.83671.09. [PubMed: 14758136]

44. Huang J, Rizk N, Park B, et al.: Recent clinical experience with multimodality therapy in thymic carcinoma. J Clin Oncol 2007, ASCO Annual Meeting Proceedings; 25: [Abstract 18003].

45. Ciccone AM, Rendina EA: Treatment of recurrent thymic tumors. Semin Thorac Cardiovasc Surg 2005, 17:27-31. doi:10.1053/j.semtcvs.2004.12.004. [PubMed: 16104358]

46•. Loehrer PJ, Wang W, Johnson DH, Ettinger DS: Octreotide alone or with prednisone in patients with advanced thymoma and thymic carcinoma. An Eastern Cooperative Oncology Group Phase II Trial. J Clin Oncol 2004, 22:293-299. doi:10.1200/JCO.2004.02.047. [PubMed: 14722038] 
47 - Loehrer PJ, Yiannoutsos CT, Dropcho S, et al.: A phase II trial of pemetrexed in patients with recurrent thymoma or thymic carcinoma. J Clin Oncol 2006, ASCO Annual Meeting Proceedings; 24: [Abstract 7079].

48. Umemura S, Segawa Y, Fujiwara K, et al.: A case of recurrent metastatic thymoma showing a marked response to paclitaxel monotherapy. Jpn J Clin Oncol 2002, 32(7):262-265. doi:10.1093/ jjco/hyf059. [PubMed: 12324577]

49. Oguri T, Achiwa H, Kato D, et al.: Efficacy of docetaxel as a second-line chemotherapy for thymic carcinoma. Chemotherapy 2004, 50:279-282. doi:10.1159/000082626. [PubMed: 15608443]

50•. Lucchi M, Basolo F, Mussi A: Surgical treatment of pleural recurrence from thymoma. Eur J Cardiothorac Surg 2008, 33:707-711. doi:10.1016/j.ejcts.2008.01.015. [PubMed: 18282761]

51. Sugie C, Shibamoto Y, Ikeya-Hashizuma C, et al.: Invasive thymoma: postoperative mediastinal irradiation, and low-dose entire hemithorax irradiation in patients with pleural dissemination. $\mathrm{J}$ Thorac Oncol 2008, 3:75-81. doi:10.1097/JTO.0b013e31815e8b73. [PubMed: 18166844]

52. DeBree E, van Ruth S, Baas P, et al.: Cytoreductive surgery and intraoperative hyperthermic intrathoracic chemotherapy in patients with malignant pleural mesothelioma or pleural metastases of thymoma. Chest 2002, 121:480-487. doi:10.1378/chest.121.2.480. [PubMed: 11834661] 

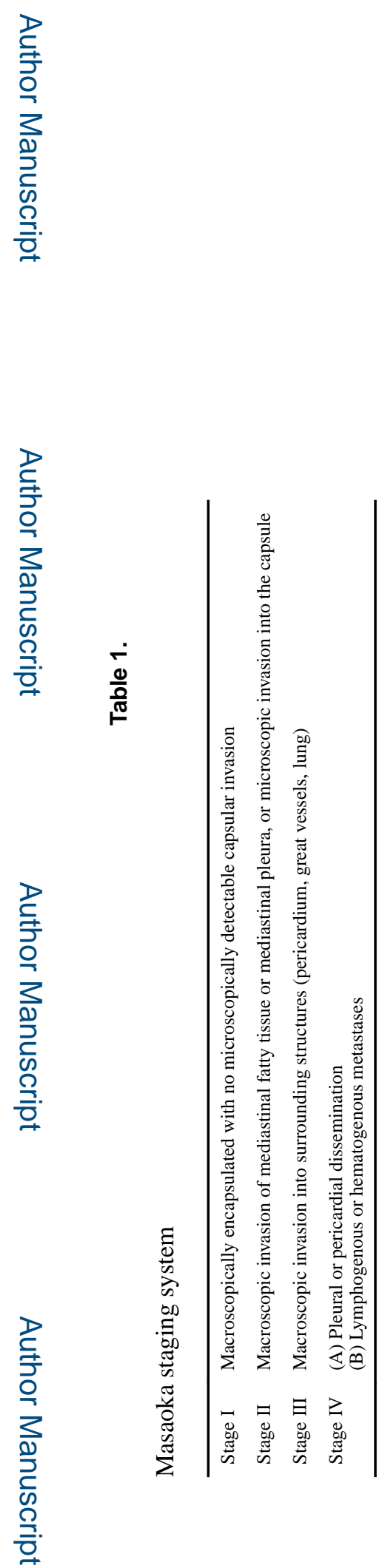

Curr Treat Options Oncol. Author manuscript; available in PMC 2019 November 13. 


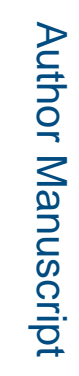

疍

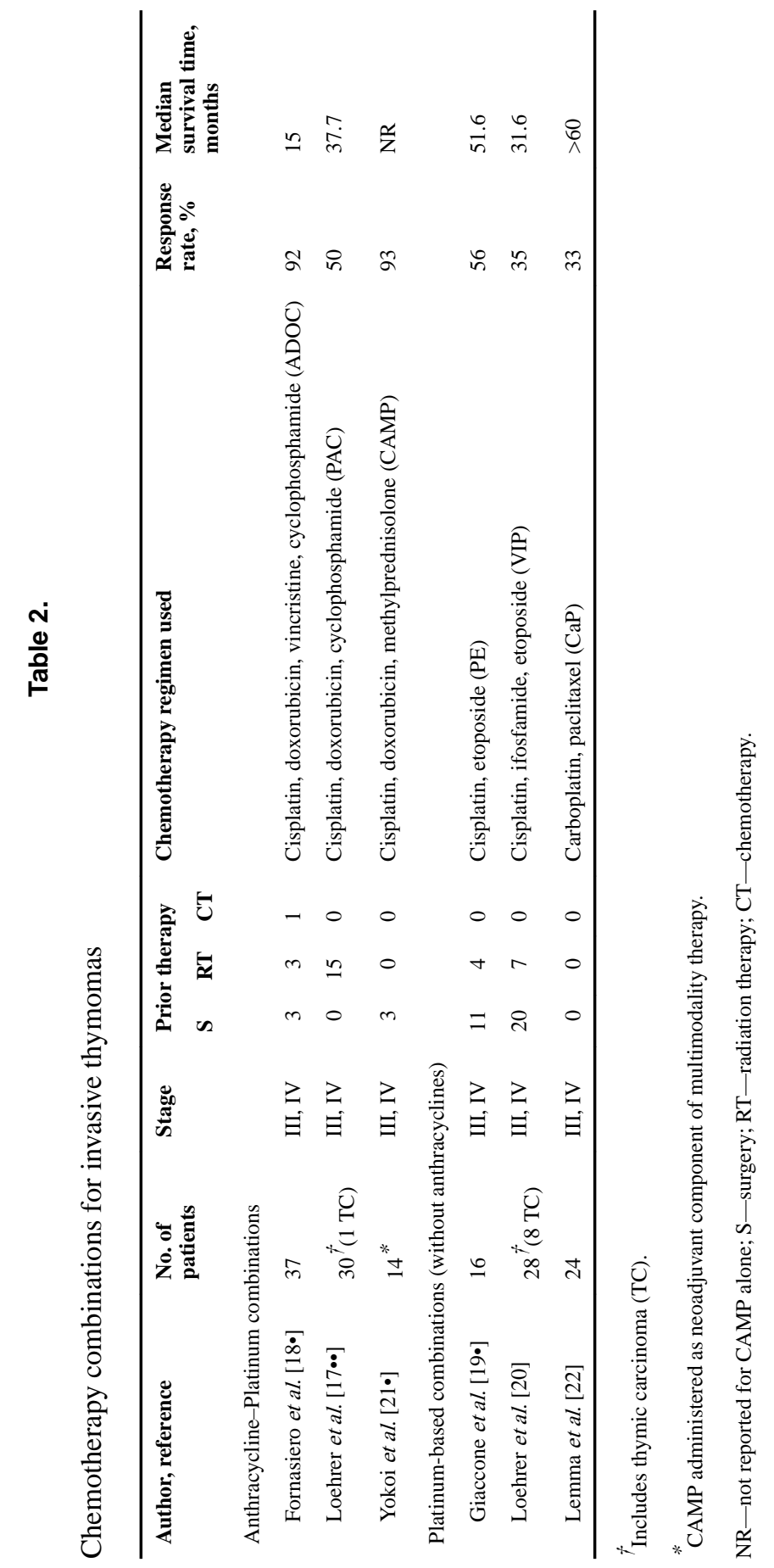

Curr Treat Options Oncol. Author manuscript; available in PMC 2019 November 13. 


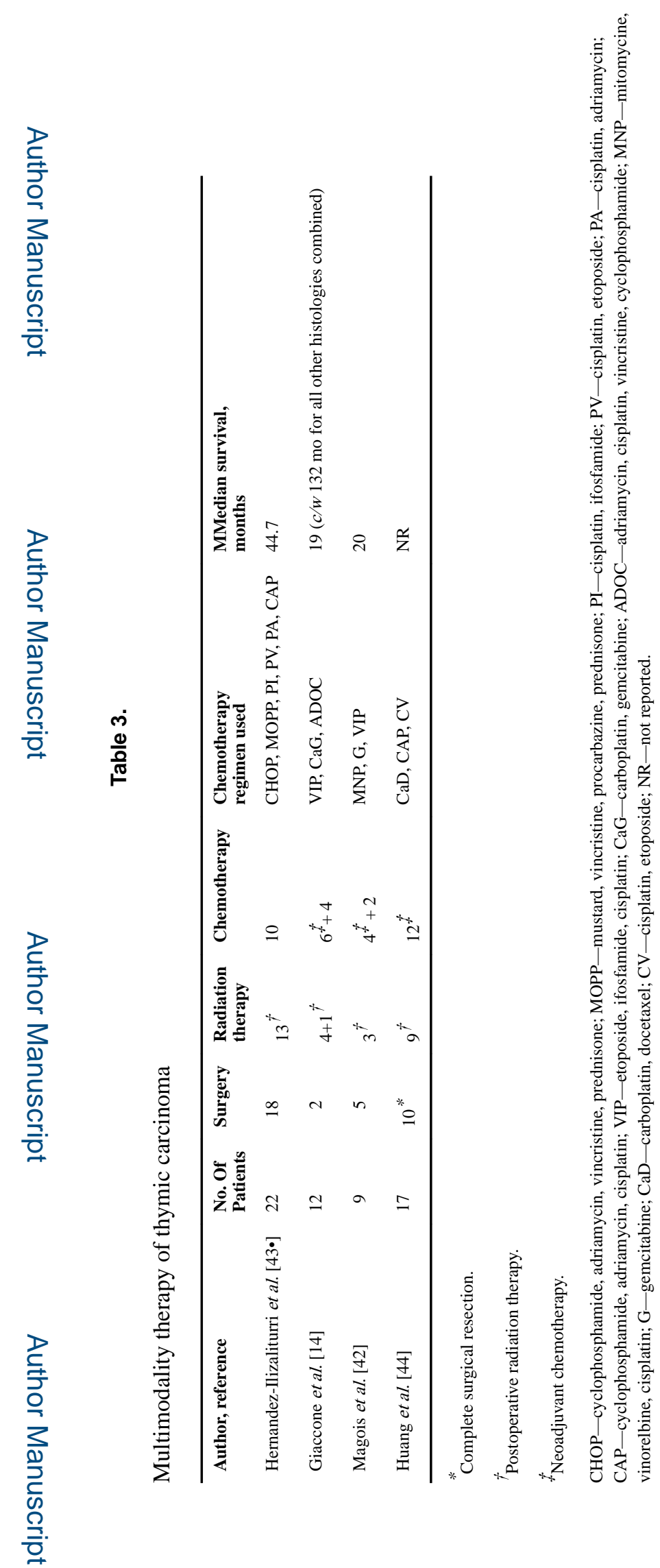

Curr Treat Options Oncol. Author manuscript; available in PMC 2019 November 13. 


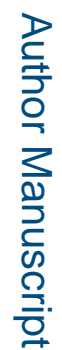

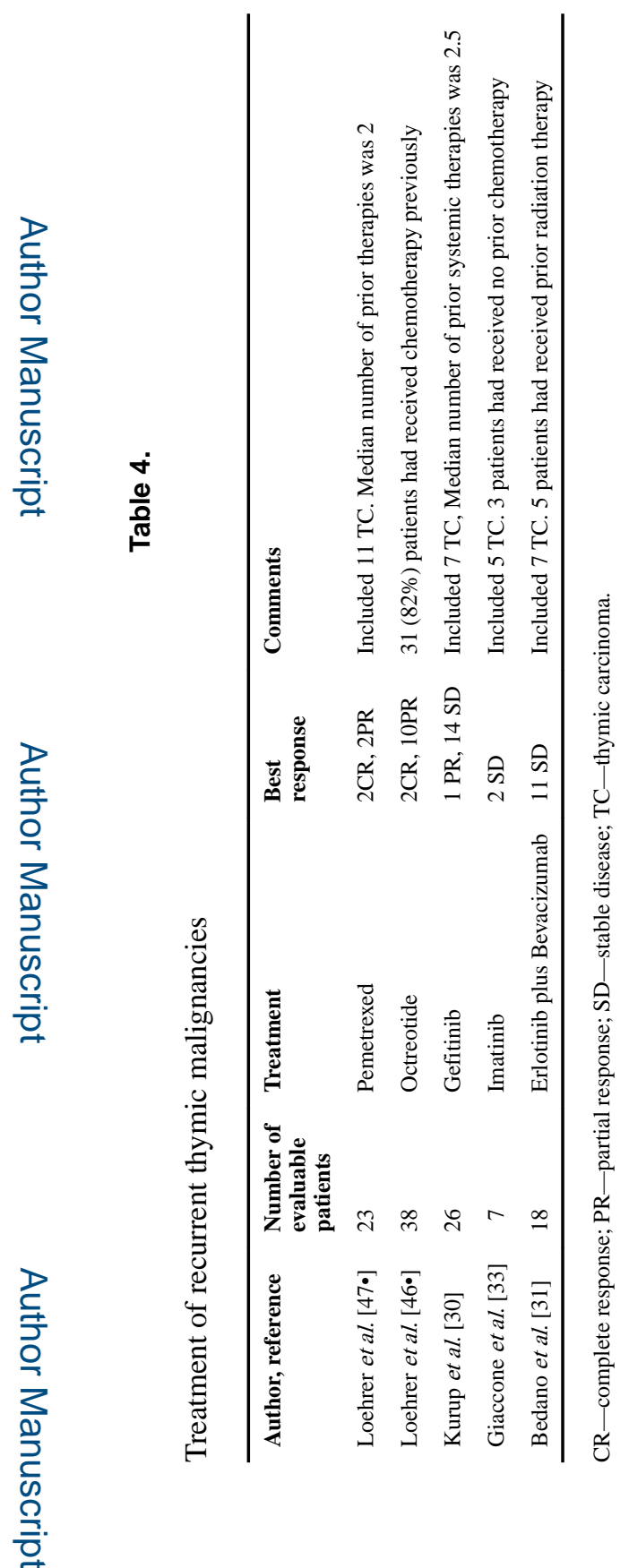

Curr Treat Options Oncol. Author manuscript; available in PMC 2019 November 13. 